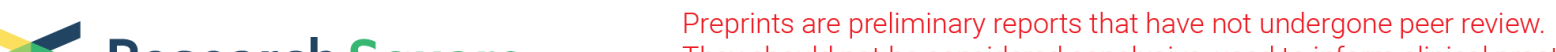 $\begin{array}{ll}\text { Research Square } & \text { They should not be considered conclusive, used to inform clinical practice, } \\ \text { or referenced by the media as validated information. }\end{array}$
}

\section{Inhibition of fibroblast growth factor-inducible 14 (Fn14) attenuates experimental tubulointerstitial fibrosis and profibrotic factor expression of proximal tubular epithelial cells}

Mai Luo

Second Affiliated Hospital of Xi'an Jiaotong University

Mengmeng Liu

Second Affiliated Hospital of Xi'an Jiaotong University

Wei Liu

Second Affiliated Hospital of Xi'an Jiaotong University

Xiao Cui

Second Affiliated Hospital of Xi'an Jiaotong University

Siyue Zhai

Second Affiliated Hospital of Xi'an Jiaotong University

Hanjiang Gu

Second Affiliated Hospital of Xi'an Jiaotong University

Huixia Wang

Second Affiliated Hospital of Xi'an Jiaotong University

Kunyi Wu

Second Affiliated Hospital of Xi'an Jiaotong University

Wen Zhang

Engineering University of PAP,China

Ke Li

Second Affiliated Hospital of Xi'an Jiaotong University

Yumin Xia ( $\square$ xiayumin1202@126.com)

Second Affiliated Hospital of Xi'an Jiaotong University

\section{Research Article}

Keywords: Fibroblast growth factor-inducible 14 (Fn14), Proximal tubular epithelial cell, Renal fibrosis, Tumor necrosis factor-like weak inducer of apoptosis (TWEAK), Unilateral ureteral obstruction

Posted Date: December 9th, 2020 
DOI: https://doi.org/10.21203/rs.3.rs-117045/v1

License: (c) (1) This work is licensed under a Creative Commons Attribution 4.0 International License. Read Full License 


\section{Abstract}

As a proinflammatory cytokine, tumor necrosis factor-like weak inducer of apoptosis (TWEAK) participates in the progression of renal fibrosis by engaging its receptor, fibroblast growth factor-inducible 14 (Fn14). However, the effect of Fn14 inhibition on tubular epithelial cell-mediated tubulointerstitial fibrosis remains unclear. This study was designed to elucidate the role of TWEAK/Fn14 interaction in the development of experimental tubulointerstitial fibrosis as well as the protective effect of Fn14 knockdown on proximal tubular epithelial cells. A murine model of unilateral ureteral obstruction was constructed in both wild-type and Fn14-deficient BALB/c mice, followed by observation of the tubulointerstitial pathologies. Fn14 deficiency ameliorated the pathological changes, including inflammatory cell infiltration and cell proliferation, accompanied by reduced production of profibrotic factors and extracellular matrix deposition. In vitro experiments showed that TWEAK dose-dependently enhanced the expressions of collagen I, fibronectin, and a-smooth muscle actin in proximal tubular epithelial cells. Interestingly, TWEAK also upregulated the expression levels of Notch1/Jagged1. Fn14 knockdown and Notch/Jagged 1 inhibition also mitigated the effect of TWEAK on these cells. In conclusion, TWEAK/Fn14 signals contributed to tubulointerstitial fibrosis by acting on proximal tubular epithelial cells. Fn14 inhibition might be a therapeutic strategy for protecting against renal interstitial fibrosis.

\section{Introduction}

Tumor necrosis factor (TNF)-like weak inducer of apoptosis (TWEAK) is a member of the TNF family and acts by binding receptor fibroblast growth factor-inducible 14 (Fn14). TWEAK/Fn14 signaling participates in the progression of renal fibrosis (1-3). Fn14 is typically weakly expressed in normal tissues but dramatically upregulated in damaged tissue, including acute and chronic kidney injury $(1,2,4,5)$. TWEAK is predominantly expressed by immune cells, such as macrophages (3), which accumulate during renal inflammation and play important roles in fibrosis (6). Excessive and persistent activation of TWEAK/Fn14 signaling promotes tissue fibrosis by recruiting inflammatory cell infiltration, production of multiple cytokines and interstitial matrix, and proliferation of resident cells that help repair the injured tissue $(7,8)$. These findings indicate that TWEAK/Fn14 interaction is instrumental in the progression of renal fibrosis.

Renal interstitial fibrosis is the final common outcome of chronic kidney diseases, characterized by myofibroblast activation and excessive accumulation of extracellular matrix. In the early stages of this process, injured proximal tubular epithelial cells (PTECs) dedifferentiate to attempt cell repair, stimulate inflammation, and recruit myofibroblasts (9). This increased tubular transport workload exceeds the energy-generating capacity, eventually leading to abnormal metabolism and fibrogenic responses (9). Epithelial-mesenchymal transition and inflammatory infiltration may subsequently occur, triggering a vicious cycle of local injury, misdirected repair, and tissue fibrosis. Activated PTECs synthesize extracellular matrix, including collagen I and fibronectin, and exhibit phenotypic changes involving overexpression of a-smooth muscle actin (a-SMA) $(10,11)$. Thus, these changes in PTECs reflect the progression of tubulointerstitial fibrosis. 
Fn14 is expressed in mesangial cells, podocytes, endothelial cells, and tubular epithelial cells in the kidneys (3). TWEAK promotes the expression of proinflammatory cytokines in these cells, including interferon gamma-induced protein 10 (IP-10), monocyte chemotactic protein-1 (MCP-1), and regulated upon activation normal T cell expressed and secreted (RANTES), which have been involved in lupus nephritis (3). Inhibition of TWEAK/Fn14 signaling exerts a protective role in some kidney diseases. Previously, we found that the neutralizing antibody targeting TWEAK significantly decreased glomerular immunoglobulin deposition, local macrophage infiltration, and tubulointerstitial fibrosis in nephrotoxic serum nephritis (12). Moreover, Fn14 deficiency preserves the filtration barrier and ameliorates spontaneous lupus nephritis, including attenuating interstitial inflammation (13). Therefore, we hypothesized that Fn14 deficiency also decreases tubulointerstitial fibrosis and protects PTECs from fibrotic phenotype changes. This study was conducted to explore the effect of Fn14 inhibition on a murine unilateral ureteral obstruction (UUO) model and PTECs in vitro.

\section{Materials And Methods}

Animal experiments. Both wild-type and Fn14-deficient BALB/c mice were used in this study. Fn14 deficiency was generated in mice by using the clustered, regularly interspaced, short palindromic repeats (CRISPR)/CRISPR-associated (Cas) 9 method (14). Mice were routinely housed the specific-pathogenfree facility of the Medical Animal Center of Xi'an Jiaotong University. Eight-week-old male mice were used in the experiments. Mice were randomly divided into the sham operation group of $\mathrm{Fn}_{14}{ }^{+/+}$mice, the sham operation group of $\mathrm{Fn} 14^{-/-}$mice, the UUO operation group of $\mathrm{Fn}_{14}^{+/+}$mice, and the UUO operation group of $\mathrm{Fn}_{14}{ }^{-/-}$mice. Mice were intraperitoneally anesthetized with pentobarbital sodium $(75 \mathrm{mg} / \mathrm{kg})$. For the UUO operation, the left ureter was isolated through a median abdominal incision and ligated with a 4 - 0 silk suture (15). Sham-operated animals underwent the same surgical procedures, except that the left ureter was operated without ligation. Mice were euthanized on days 3, 5 or 7 after surgery. The medical ethics committee of the Second Affiliated Hospital of Xi'an Jiaotong University approved all animal study procedures.

Histological evaluation and immunohistochemistry. Renal tissues were routinely processed for paraffin sectioning, then further stained with hematoxylin and eosin (H\&E), periodic-acid Schiff (PAS), Masson trichrome or Sirius red. All sections were scanned using a Nano Zoomer 2.0 HT slide scanner (Hamamatsu Photonics, Hamamtsu, Japan), then analyzed using the NDP.view2 software (Hamamatsu Photonics). Six to 8 cortex fields were randomly selected per section. Two pathologists blinded to the groupings evaluated the histological changes. Tubular injury (including atrophy, casts, dilatation, and inflammatory infiltration) was scored on the H\&E- and PAS-stained sections using a six-point scale in which $0,1,2,3,4$, and 5 indicated normal, very little, very mild, mild, moderate, and severe histological lesions, respectively (16). Renal interstitial fibrosis was assessed on Sirius red and Masson trichromestained sections, and positive signals were quantified using Image Pro-Plus 6.0 software (Media Cybernetics, Rockville, MD, USA). 
Paraffin sections were routinely processed for immunohistochemistry. After blocking with endogenous peroxidase blocking buffer (Beyotime Co., Shanghai, China), rabbit anti-collagen I, a-SMA, fibronectin, CD45, F4/80 or Ki67lgG (Abcam, Cambridge, MA, USA) were used as the primary antibodies. Horseradish peroxidase-conjugated goat anti-rabbit IgG was the secondary antibody (Abcam). 3,3'-Diaminobenzinechromogen substrates was used for signal development (CWbio, Beijing, China). To evaluate collagen I, fibronectin, and a-SMA expression, ten fields per section were selected randomly, then quantified using Image Pro-Plus 6.0 software. Two pathologists counted the $\mathrm{CD}_{4} 5^{+}, \mathrm{F} 4 / 80^{+}$and $\mathrm{Ki} 67^{+}$cells in a singleblinded manner.

Cell culture. Human PTECs (HK2) were purchased from China Center for Type Culture Collection (CCTCC, Wuhan, China), and cultured in Hyclone Dulbecco's modified Eagle's medium (GE Healthcare, Port Washington, NY, USA) containing 10\% fetal bovine serum and 1\% penicillin/streptomycin (Gibco, Waltham, MA, USA). Before the stimulation assays, HK2 cells were starved in $1 \%$ fetal bovine serumsupplemented medium for $12 \mathrm{~h}$. Recombinant human TWEAK (0-250 ng/ml; Peprotech, Cranbury, NJ, USA) was then added for $24 \mathrm{~h}$.

In some experiments, HK2 cells were transfected with Fn14 or control siRNA before the stimulation assays (17). Cells grown in six-well plates were added with the siRNA oligomer and Lipofectamine 3000 reagent complexes (Life Technologies, Carlsbad, CA, USA). The Fn14 siRNA sequences were siRNA1, GCAGGAGAGAGAAGUUCACTT (sense) and GUGAACUUCUCUCUCCUGCTT (antisense) and siRNA2, CACUGACUAAGGAACUGCATT (sense) and UGCAGUUCCUUAGUCAGUGTT (antisense). The transfection efficiency was verified via quantitative real-time polymerase chain reaction (qRT-PCR) and Western blotting (Fig.s1). The inhibitor of Notch1/Jagged1, DAPT $\left(\mathrm{C}_{23} \mathrm{H}_{26} \mathrm{~F}_{2} \mathrm{~N}_{2} \mathrm{O}_{4} ; \mathrm{Abcam}\right)$, was added $1 \mathrm{~h}$ before TWEAK stimulation.

Semi-quantitative RT-PCR. Total RNA was extracted from fresh tissue samples or cell cultures using TRIzol reagent (Invitrogen, Grand Island, NY, USA). cDNA was synthesized using PrimeScript ${ }^{\mathrm{TM}}$ RT Master Mix (Takara Bio, Shiga, Japan). qRT-PCR was performed on the ABI StepOne System (Applied Biosystems, Carlsbad, CA, USA) using SYBR green master mix as the fluorescent dye (Takara). The glyceraldehyde-3-phosphate dehydrogenase (GAPDH) gene was used as the endogenous control. The PCR primer sequences were synthesized by Sangon Biotech (Shanghai, China; Table 1). The mRNA expression levels were calculated using the $2-\Delta \Delta C$ t method, then normalized to the GAPDH values. 
Table 1

Sequences of primers

\begin{tabular}{|c|c|}
\hline Primer & Sequence \\
\hline \multirow[t]{2}{*}{ Human a-SMA } & Forward: 5'-GCTGACAGAGGCACCACTGAAC-3' \\
\hline & Reverse: 5'-AGTCACACCATCTCCAGAGTCCAG-3' \\
\hline \multirow[t]{2}{*}{ Human collagen } & Forward: 5'-GCGAGAGCATGACCGATGGATTC-3' \\
\hline & Reverse: 5'-GCCTTCTTGAGGTTGCCAGTCTG-3' \\
\hline \multirow[t]{2}{*}{ Human fibronectin } & Forward: 5'-ATGCAACGATCAGGACACAAGGAC-3' \\
\hline & Reverse: 5'-TGCCTCTCАСАСТTССАСТСТСС-3' \\
\hline \multirow[t]{2}{*}{ Human Fn14 } & Forward: 5'-CTCTGAGCCTGACCTTCGTG-3' \\
\hline & Reverse: 5'-GGGGGCACATTGTCACTGGA-3' \\
\hline \multirow[t]{2}{*}{ Human GAPDH } & Forward: 5'-CAGGAGGCATTGCTGATGAT-3' \\
\hline & Reverse: 5'-GAAGGCTGGGGCTCATTT-3' \\
\hline \multirow[t]{2}{*}{ Human Jagged1 } & Forward: 5'-TCAGGCAGAAGCAGAGGTAGGC-3' \\
\hline & Reverse: 5'-TGTGGCTTGGATCTGTTGCTTGG-3' \\
\hline \multirow[t]{2}{*}{ Human Notch1 } & Forward: 5'-TGCGAGACCAACATCAACGAGTG-3' \\
\hline & Reverse: 5'-ACGTTGTTGGTGGTGTTGTCCTC-3' \\
\hline \multirow[t]{2}{*}{ Mouse a-SMA } & Forward: 5'-GCTGGTGATGATGCTCCCA-3' \\
\hline & Reverse: 5'-GCCCATTCCAACCATTACTCC-3' \\
\hline \multirow[t]{2}{*}{ Mouse collagen I } & Forward: 5'-TGACTGGAAGAGCGGAGAGT-3' \\
\hline & Reverse: 5'-GTTCGGGCTGATGTACCAGT-3' \\
\hline \multirow[t]{2}{*}{ Mouse CTGF } & Forward: 5'-CTTCTGCGATTTCGGCTCC-3' \\
\hline & Reverse: 5'-TACACCGACCCACCGAAGA-3' \\
\hline \multirow[t]{2}{*}{ Mouse fibronectin } & Forward: 5'-ACAGTCCAGCAAGCAGCAAGC-3' \\
\hline & Reverse: 5'-TGGTGGTCACTCTGTAGCCTGTC-3' \\
\hline \multirow[t]{2}{*}{ Mouse GAPDH } & Forward: 5'-TGTGTCCGTCGTGGATCTGA-3' \\
\hline & Reverse: 5'-TTGCTGTTGAAGTCGCAGGAG-3' \\
\hline Mouse IL-6 & Forward: 5'-TGATGGATGCTACCAAACTGGA-3' \\
\hline
\end{tabular}




\begin{tabular}{|ll|}
\hline Primer & \multicolumn{1}{|c|}{ Sequence } \\
\hline Mouse IP-10 & Reverse: 5'-CTGAAGGACTCTGGCTTTGTCT-3' \\
\hline Mouse MCP-1 & Forward: 5'-GGCCATAGGGAAGCTTGAAA-3' \\
\hline & Reverse: 5'-CAGACATCTCTGCTCATCATTCT-3' \\
\hline Mouse TNF-a & Forward: 5'-GGCTCAGCCAGATGCAGTTA-3' \\
\hline & Reverse: 5'-ATTTGGTTCCGATCCAGGTT-3' \\
\hline $\begin{array}{l}\text { Abbreviations: a-SMA, a-smooth muscle actin; CTGF, connective tissue growth factor; Fn14, fibroblast } \\
\text { growth factor inducible 14; GAPDH, glyceraldehyde 3-phosphate dehydrogenase; IP-10, interferon } \\
\text { gamma-induced protein 10; MCP-1, monocyte chemotactic protein-1; TNF-a, tumor necrosis factor }\end{array}$ \\
\hline
\end{tabular}

Western blot analysis. Fresh tissue or cell cultures were ground with a homogenizer in RIPA lysis buffer with protease inhibitors (HEART, Xi'an, China). The protein samples were $20 \mu \mathrm{g}$ per well. Proteins were separated by sodium dodecyl sulfate-polyacrylamide gel electrophoresis (HEART), then transferred onto polyvinylidene difluoride membranes (Millipore, Billerica, MA, USA). After blocking with $5 \%$ nonfat milk, the membranes were incubated with primary antibodies targeting GAPDH (Cell Signaling, Danvers, MA, USA), collagen I (Cell Signaling), a-SMA (Abcam), fibronectin (Abcam), Notch1 (Abcam) or Jagged1 (Abcam). The horseradish peroxidase-conjugated secondary antibodies were purchased from Proteintech (Wuhan, China). The signals were detected with an electrochemiluminescence kit (Millipore). The band intensities were quantified using ImageJ software (National Institutes of Health, Bethesda, MD, USA).

Enzyme-linked immunosorbent assay (ELISA). ELISAs were performed with tissue lysates. Fresh tissue was ground in phosphate-buffered saline for the protein lysates. All lysate samples were normalized according to the original tissue weight. The commercial immunoassay kits (targeting mouse IL- 6 and TNF-a) were purchased from BD Bioscience (San Jose, CA, USA). The commercial kits (targeting mouse MCP-1) were purchased from R\&D Systems (Minneapolis, MN, USA). The commercial kits (targeting mouse IP-10) were purchased from Elabscience Biotechnology (Wuhan, China). The protocols were performed per the manufacturer's instructions.

Statistical analysis. Data are expressed as the means \pm SEM and were statistically analyzed using SPSS software (SPSS Inc., Chicago, IL, USA). Bonferroni multiple comparison tests and analysis of variance were used for multiple comparisons. Statistical differences were considered significant at $P<0.05$.

\section{Results}


Fn14 deficiency ameliorated tubulointerstitial fibrosis in UUO mouse models. Tubulointerstitial changes were observed at different time points after UUO. PAS and H\&E staining showed no histological abnormalities in the sham groups (Fig. 1a-d). However, severe morphological injury, including renal tubular expansion, loss of renal tubular epithelium, inflammatory cell infiltration, mesangial expansion, and cast formation were observed in the operated groups. Fn14-deficient mice had lower histological scores for tubular injury on days 5 and 7 (Fig. 1a-d). Sirius red and Masson trichrome staining of the extracellular matrix accumulation showed that Fn14-deficient mice had lower scores for tubulointerstitial fibrosis on days 3 and 7 compared with those of the wild-type mice (Fig. 1e-h). Fn14 deficiency ameliorated tubular injury, including fibrosis, in the UUO mouse model.

Fn14 deficiency reduced profibrotic factor production in the kidneys. The early stages of UUO are characterized by tubulointerstitial inflammation (9). The expression levels of inflammatory cytokines, including MCP-1 and IP-10, were assessed via qRT-PCR and ELISA. Fn14-deficient mice had lower mRNA and protein expression levels of MCP-1 and IP-10 on days 3 and 5 as well as decreased expression levels of interleukin (IL)-6 and TNF-a (Fig. 2).

The expression levels of fibrotic markers (collagen I and fibronectin) and myofibroblast activation markers (a-SMA and connective tissue growth factor (CTGF)) were determined via qRT-PCR and Western blotting. The Fn14-deficient mice had lower mRNA expression levels of collagen I, a-SMA, and CTGF than did the wild-type mice after UUO (Fig. 3a-d). On day 7, the Fn14-deficient mice had reduced collagen I, fibronectin, and a-SMA expressions (Fig. 3e, f). Moreover, collagen I and fibronectin production increased time-dependently in the wild-type mice (Fig. 3g, h).

Collagen I, fibronectin, and a-SMA expressions were further detected in the kidney tissue via immunohistochemistry, and their expressions were significantly higher in the wild-type mice than in the Fn14-deficient mice on day 7 (Fig. 4). Thus, Fn14 deficiency reduced fibrotic marker expression in the kidneys of the UUO model mice.

Fn14 deficiency inhibited inflammatory cell recruitment. Inflammatory responses are initial factors in the development of renal fibrosis and involve leukocytes such as macrophages (6). Immunohistochemistry showed that $C D 45^{+}$leukocytes were intensively recruited on day 7 in the wild-type mice but less detected in the Fn14-deficient mice (Fig. 5a, b). Similarly, F4/80+ macrophage infiltration was attenuated in the Fn14-deficient mice on day 7 (Fig. $5 \mathrm{c}$, d). Cell proliferation is subsequent to tissue inflammation in UUO model mice (18). More $\mathrm{Ki} 67^{+}$proliferative cells were observed in the kidneys of the wild-type mice than in those of the Fn14-knockout mice on day 7 (Fig. 5e, f). Therefore, Fn14 deficiency attenuated inflammatory cell infiltration in the kidneys after UUO.

TWEAK promoted fibrotic marker production in PTECs. PTECs play a crucial role in the progression of tubulointerstitial fibrosis (10). To investigate the effects of TWEAK/Fn14 interaction on fibrotic marker synthesis, HK2 cells were stimulated by human recombinant TWEAK $(0-250 \mathrm{ng} / \mathrm{ml})$. TWEAK dosedependently increased the mRNA expression levels of collagen I, fibronectin, and a-SMA (Fig. 6a-c). 
Similarly, recombinant TWEAK promoted protein expression of these molecules (Fig. 6d, e). However, Fn14 siRNA transfection in HK2 cells tempered this TWEAK effect on the mRNA and protein expression levels of collagen I, fibronectin, and a-SMA (Fig. 6f-h and Fig.S1).

TWEAK activates the Notch1/Jagged1 signaling pathway in HK2 Cells. Notch1/Jagged1 signaling is reported to be involved in interstitial fibrosis in kidney diseases (11). We investigated the activity of Notch1/Jagged1 signals in response to TWEAK stimulation in HK2 cells, and exogenous TWEAK (0$250 \mathrm{ng} / \mathrm{ml}$ ) enhanced the Notch1 and Jagged1 mRNA expression levels (Fig. 7a, b). Consistently, protein expression was upregulated in these cells upon TWEAK stimulation (Fig. 7c, d), and pretransfection of Fn14 siRNA mitigated the effect of TWEAK on HK2 cells (Fig. 7e-g).

DAPT is a specific inhibitor of the Notch1 signaling pathway (19). DAPT decreased the enhancement effect of TWEAK on the mRNA expression of profibrotic factors, collagen I, fibronectin, and a-SMA, as well as Notch1/Jagged 1 molecules in HK2 cells (Fig. 8a, b). Consistently, DAPT mitigated the effect of TWEAK on the protein expression of profibrotic factors and Notch1/Jagged1 molecules in these cells (Fig. 8c-f). Therefore, TWEAK-induced fibrotic outcomes depended on the Notch1/Jagged1 signaling pathway in HK2 cells.

\section{Discussion}

In this study, Fn14 deficiency ameliorated UUO-induced renal interstitial fibrosis. The protective role of Fn14 deficiency involves decreased extracellular matrix accumulation and myofibroblast marker expression. In addition, Fn14 deficiency significantly reduced the inflammatory cell infiltration and proinflammatory cytokine production in the UUO mouse model. Furthermore, TWEAK/Fn14 interaction enhanced the expression levels of collagen I, fibronectin, and a-SMA in cultured PTECs, and the Notch1/Jagged1 inhibitor mitigated the effect of TWEAK on cells. Therefore, the TWEAK/Fn14 signals participate in UUO-induced interstitial fibrosis in mice, involving Notch1/Jagged1 signal activation in PTECs.

Previous studies showed that TWEAK/Fn14 signaling is activated in classic experimental animal models of acute kidney injury, lupus nephritis, and spontaneous chronic kidney disease $(3,4,13,20,21)$. Both Fn14 and TWEAK are highly upregulated in UUO-induced kidney injury with fibrosis (21). TWEAK deficiency leads to delayed renal interstitial fibrosis in mice after UUO (1). We recently found that inhibiting the TWEAK/Fn14 pathway attenuated renal disease in nephrotoxic serum nephritis, which mimics the histological features of lupus nephritis (12). Here, we report for the first time that Fn14 deficiency attenuated tubulointerstitial fibrosis after UUO. Our results were consistent with previous findings in verifying the contributive role of TWEAK/Fn14 signaling in interstitial fibrosis in kidney injury. Fn14 upregulation is relatively restricted to injured tissues or organs. Hence, inhibiting Fn14 but not TWEAK more specifically reduced renal damage. Second, different from previous reports, TWEAK directly acted on PTECs by enhancing profibrotic factor expression. Because PTECs are central in 
tubulointerstitial injury processes, our findings strongly suggest that TWEAK/Fn14 activation plays an important role in tubulointerstitial fibrosis.

UUO is a classic model of renal interstitial fibrosis used to study the mechanisms of multiple kidney diseases. In addition to inflammatory cell infiltration, extracellular matrix deposition characterizes the renal histology of UUO (22). In this study, Fn14 deficiency decreased the leukocyte and macrophage infiltration in the UUO model. Moreover, extracellular matrix deposition was significantly reduced in the Fn14-deficient strain. The expression levels of fibrotic markers (collagen I and fibronectin) and myofibroblast activation markers (a-SMA and CTGF) were also downregulated in these mice. Fibroblastmyofibroblast transdifferentiation is a major feature of renal interstitial fibrosis (23). The a-SMA ${ }^{+}$ myofibroblasts activate during fibrotic processes, synthesizing myofibroblast phenotypic markers, including CTGF. Myofibroblasts are also the predominant collagen-producing cells during fibrosis activation (2). Increased a-SMA expression in the UUO mice was reduced upon Fn14 deficiency, accompanied by reduced collagen I, fibronectin, and CTGF expressions. Thus, TWEAK participates in renal interstitial fibrosis by promoting fibroblast-myofibroblast transdifferentiation.

TWEAK induces inflammatory cytokines, such as MCP-1, IP-10, and IL-6, which are expressed by renal resident cells and exacerbate local inflammation and interstitial fibrosis $(13,24)$. TWEAK also inhibits expression of suppressor of cytokine signaling 1 in kidney cells, which negatively regulates tissue fibrosis and triggers transcription of genes that encode profibrotic factors $(25,26)$. In this study, MCP-1, IP- 10 , IL6 , and TNF-a increased time-dependently in the kidneys of a UUO model, while Fn14 deficiency suppressed the production of these cytokines. Leukocytes, such as lymphocytes and macrophages, are the main cells that secrete inflammatory cytokines and are suggested to be involved in the development of renal interstitial fibrosis $(3,27)$. Our experiments showed that leukocyte and macrophage infiltration was reduced in the Fn14-deficient UUO model. In addition, proliferative $\mathrm{Ki} 67^{+}$cells in the kidneys were reduced in the same strain. Therefore, inhibiting downstream cytokines, inflammatory cell infiltration, and subsequent cell proliferation mediated the protective effect of Fn14 deficiency in UUO mice.

PTECs are the major resident cells in the kidneys and play a crucial role in the fibrotic progression of kidney diseases (28). PTECs undergo epithelial-mesenchymal transition and produce extracellular matrix components (28). Hence, we investigated the in vitro effect of TWEAK on PTECs (HK2 cells). TWEAK enhanced the collagen I and fibronectin synthesis dose-dependently. Consistently, expression of a-SMA, a myofibroblast activation marker, increased with TWEAK stimulation. TWEAK was previously shown to induce renal and embryonic fibroblast proliferation via the NF-KB and Ras/ERK signaling pathways (1, 29). The Notch1/Jagged 1 signaling pathway also participates in the pathogenesis of tubulointerstitial fibrosis (30). In this study, the Notch1/Jagged1 proteins were upregulated in HK2 cells upon TWEAK stimulation. Inhibiting the Notch1/Jagged1 signaling pathway mitigated the promotion effect of TWEAK on the expressions of extracellular matrix and myofibroblast activation markers in these cells. Thus, the Notch1/Jagged1 signaling pathway mediated the direct effect of TWEAK on PTECs. 
In conclusion, Fn14 deficiency ameliorated tubulointerstitial fibrosis in a murine UUO model by suppressing inflammatory cytokine secretion and extracellular matrix accumulation. TWEAK upregulated the profibrotic factors and myofibroblast activation markers in PTECs by activating the Notch1/Jagged 1 signaling pathway. Targeting the TWEAK/Fn14 pathway may be a promising therapeutic approach for patients with renal interstitial fibrosis.

\section{Declarations}

\section{Data Availability}

The data used to support the findings of this study are available from the corresponding author upon request.

\section{Conflict of Interest}

The authors declare no conflict of interest.

\section{Funding Statement}

This study was supported by the National Natural Science Foundation of China (Project No.81874241) and the Innovation Capability Support Plan of Shaanxi Province (No.2019TD-034).

\section{Supplementary Materials}

The Supplementary Material for this article can be found online.

\section{Ethics Statement}

This study was carried out in accordance with the recommendations of the guidelines of the University Research Ethics Committee. These protocols were approved by the University Research Ethics Committee.

\section{Author Contributions}

M.L. and M.M. L participated in the design of the study, and performed most experimental work. WL, XC, $\mathrm{SZ}, \mathrm{HG}, \mathrm{HW}$, and KW carried out some experiments. WZ discussed the experimental data and contributed to the interpretation of results. $\mathrm{KL}$ and $\mathrm{YX}$ conceived and designed the study and prepared the manuscript. All the authors read and approved the final manuscript.

\section{References}

1. Ucero, A.C.et al. TNF-related weak inducer of apoptosis (TWEAK) promotes kidney fibrosis and Rasdependent proliferation of cultured renal fibroblast. Biochim Biophys Acta. 1832,1744-1755 (2013).

2. Sanz, A.B., Sanchez-Nino, M.D. \& Ortiz, A. TWEAK, a multifunctional cytokine in kidney injury. Kidney Int. 80,708-718 (2010). 
3. Chen, J.Y., Wei, L.L. \& Xia, Y.M. Roles of tumour necrosis factor-related weak inducer of apoptosis/fibroblast growth factor-inducible 14 pathway in lupus nephritis. Nephrology (Carlton). 22,101-106 (2017).

4. Weinberg, J.M. TWEAK-Fn14 as a mediator of acute kidney injury. Kidney Int. 79,151-153 (2011).

5. Mo, S.J.et al. Regulation of Fn14 stability by SCFFbxw7a during septic acute kidney injury. Am J Physiol Renal Physiol. 316, F1273-F1281 (2019).

6. Tang, P.M., Nikolic-Paterson, D.J. \& Lan, H.Y. Macrophages: versatile players in renal inflammation and fibrosis. Nat Rev Nephrol. 15,144-158(2019).

7. Burkly, L.C. TWEAK/Fn14 axis: the current paradigm of tissue injury-inducible function in the midst of complexities. Semin Immunol. 26,229-236(2014).

8. Liu, J.et al. TWEAK/Fn14 signals mediate burn wound repair. J Invest Dermatol. 139,224-234(2019).

9. Schnaper, H.W. The tubulointerstitial pathophysiology of progressive kidney disease. Adv Chronic Kidney Dis. 24,107-116(2017).

10. Gewin, L.S. Renal fibrosis: Primacy of the proximal tubule. Matrix Biol. 68-69,248-262(2018).

11. Hong, W.L.et al. Epithelial and interstitial Notch1 activity contributes to the myofibroblastic phenotype and fibrosis. Cell Commun Signal. 17,145(2019).

12. Xia, Y.M.et al. Inhibition of the TWEAK/Fn14 pathway attenuates renal disease in nephrotoxic serum nephritis. Clin Immunol. 145,108-121(2012).

13. Xia, Y.M.et al. Deficiency of fibroblast growth factor-inducible 14 (Fn14) preserves the filtration barrier and ameliorates lupus nephritis. J Am Soc Nephro. 26,1053-1070(2015).

14. Peng, L.L.et al. Fn14 deficiency ameliorates psoriasis-like skin disease in a murine model. Cell Death Dis. 9,801(2018).

15. Fierro-Fernández, M.et al. MiR-9-5p protects from kidney fibrosis by metabolic reprogramming. FASEB J. 34,410-431(2020).

16. Choudhry, N.et al. The complement factor 5 a receptor 1 has a pathogenic role in chronic inflammation and renal fibrosis in a murine model of chronic pyelonephritis. Kidney Int. 90,540554(2016).

17. Liu, Y.L.et al. TWEAK/Fn14 activation contributes to the pathogenesis of bullous pemphigoid. J Invest Dermatol. 137,1512-1522(2017).

18. Duffield, J.S. Cellular and molecular mechanisms in kidney fibrosis. J Clin Invest. 124,22992306(2014).

19. Soni, H., Matthews, A.T., Pallikkuth, S., Gangaraju, R., Adebiyi, A. Y-secretase inhibitor DAPT mitigates cisplatin-induced acute kidney injury by suppressing Notch1 signaling. J Cell Mol Med. 23,260270(2019).

20. Hotta, K.et al. Direct targeting of fibroblast growth factor-inducible 14 protein protects against renal ischemia reperfusion injury. Kidney Int. 79,179-188(2011). 
21. Gomez, I.G.et al. TWEAK-Fn14 signaling activates myofibroblasts to drive progression of fibrotic kidney disease. J Am Soc Nephrol. 27,3639-3652(2016).

22. Wang, Y.H., Zhou, Q.L., Tang, R., Huang, Y.Y., He, T. FoxM1 inhibition ameliorates renal interstitial fibrosis by decreasing extracellular matrix and epithelial-mesenchymal transition. J Pharmacol Sci. 143,281-289(2020).

23. Li, N.N., Wang, Z., Gao, F.L., Lei, Y.F., Li, Z.Z. Melatonin ameliorates renal fibroblast-myofibroblast transdifferentiation and renal fibrosis through miR-21-5p regulation. J Cell Mol Med. 24,56155628(2020).

24. Mohamed, R., Jayakumar, C., Ramesh, G. Chronic administration of EP4-selective agonist exacerbates albuminuria and fibrosis of the kidney in streptozotocin-induced diabetic mice through IL-6. Lab Invest. 93,933-945(2013).

25. Chen, J.Y.et al. Inhibition of suppressor of cytokine signaling 1 mediates the profibrotic effect of TWEAK/Fn14 signaling on kidney cells. Cell Signal. 71,109615(2020).

26. Wang, H.X., Wang, J.X., Xia, Y.M. Defective suppressor of cytokine signaling 1 signaling contributes to the pathogenesis of systemic lupus erythematosus. Front Immunol. 8,1292(2017).

27. Wang, Y.Y.et al. Macrophage-to-myofibroblast transition contributes to interstitial fibrosis in chronic renal allograft injury. J Am Soc Nephrol. 28,2053-2067(2017).

28. Gu, T.T.et al. Pterostilbene alleviates fructose-induced renal fibrosis by suppressing TGF- $\beta 1 /$ TGF- $\beta$ type I receptor/Smads signaling in proximal tubular epithelial cells. Eur J Pharmacol. 842,7078(2019).

29. Burkly, L.C. Regulation of Tissue Responses: The TWEAK/Fn14 pathway and other tnf/tnfr superfamily members that activate non-canonical NFkB signaling. Front Immunol. 6,92(2015).

30. Bielesz, B.et al. Epithelial Notch signaling regulates interstitial fibrosis development in the kidneys of mice and humans. J Clin Invest. 120,4040-4054(2010).

\section{Figures}


a
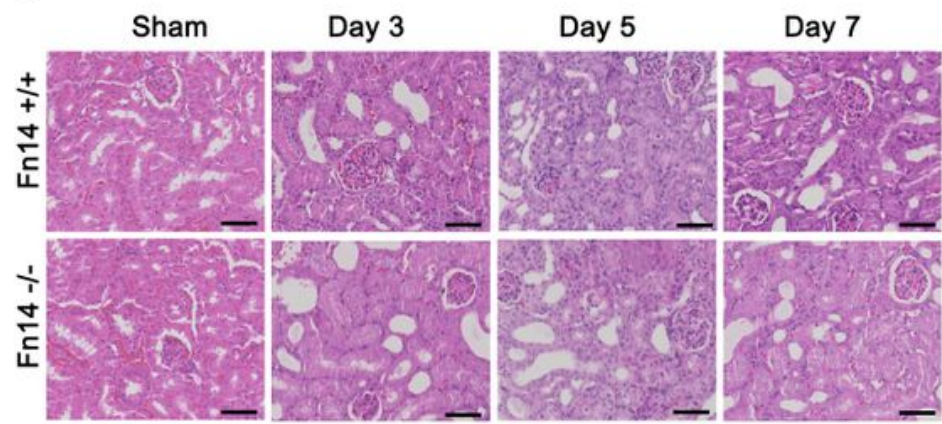

C

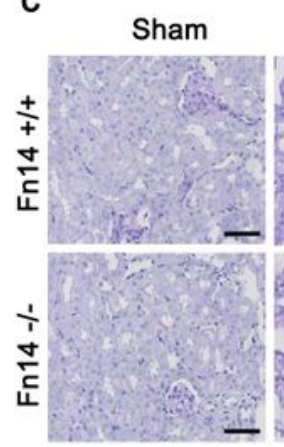

Day 3
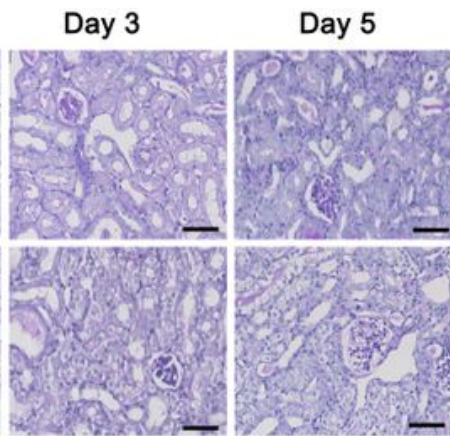

Day 7

e

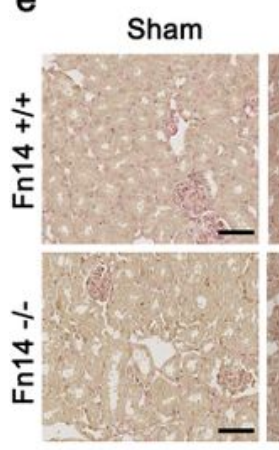

Day 3

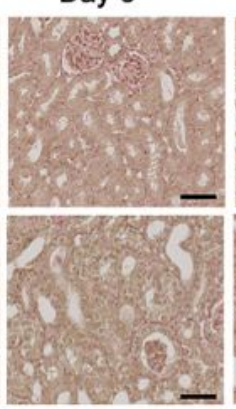

Day 5
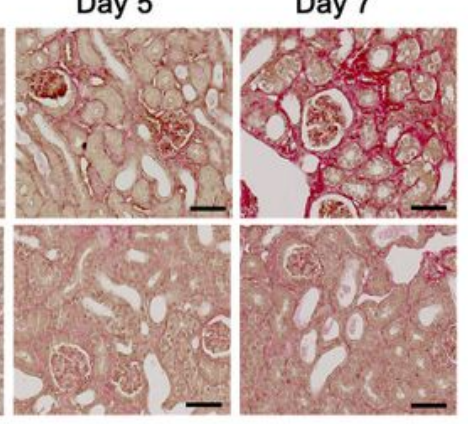

g

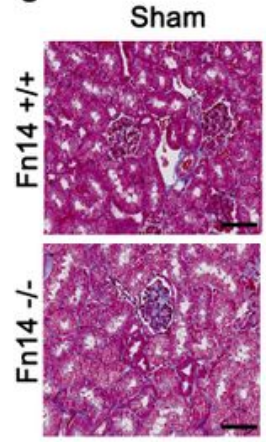

Day 3

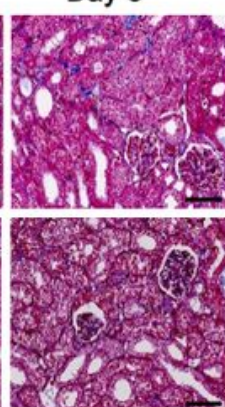

b

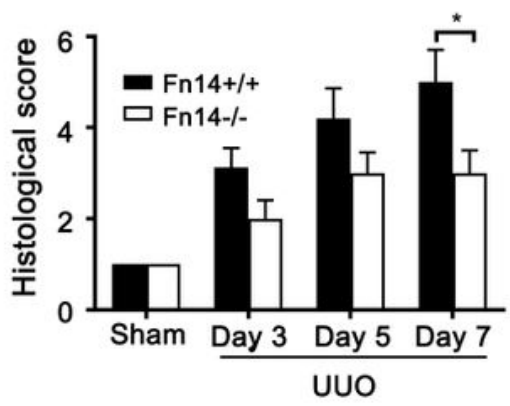

d

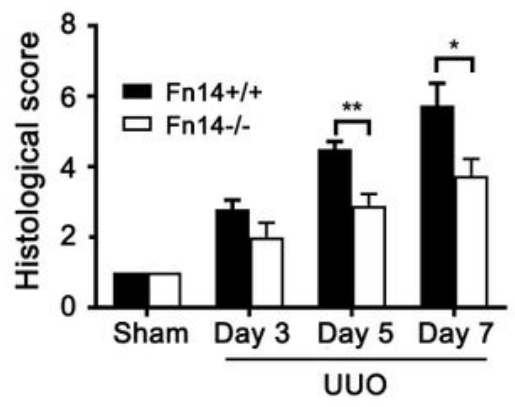

f

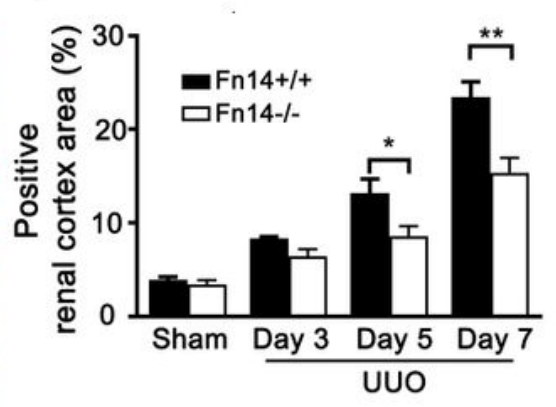

h

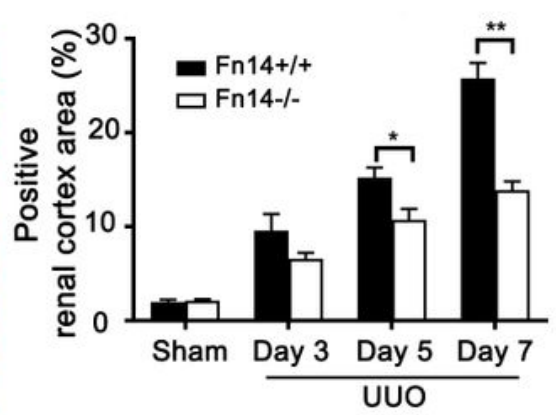

\section{Figure 1}

Fn14 deficiency ameliorated tubulointerstitial fibrosis in a UUO model. $(a, b)$ H\&E staining showing histological changes on days 0-7. (c, d) PAS staining showing similar changes on days 0-7. (e, f) Sirius red staining of the extracellular matrix on days $0-7 .(\mathrm{g}, \mathrm{h})$ Masson-trichrome staining of the extracellular matrix. The positive areas were quantified via Image-Pro Plus 6.0 software. Representative images are shown. Bar $=50 \mu \mathrm{m}$. Number of mice $=5$ per group. ${ }^{*} \mathrm{P}<0.05, * * \mathrm{P}<0.01$. 
a

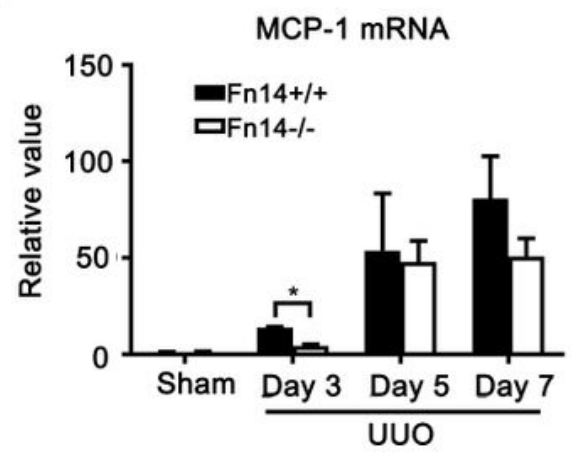

C

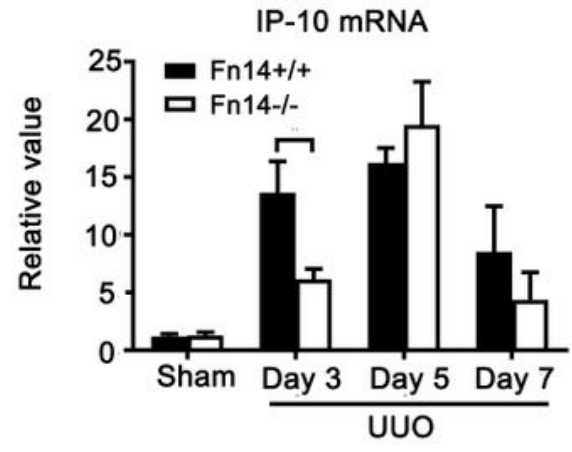

e

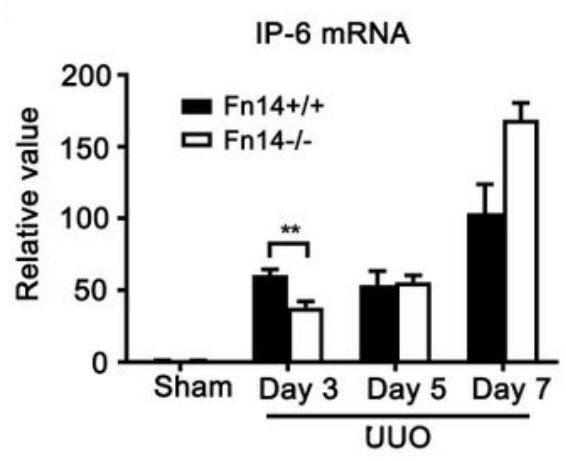

g

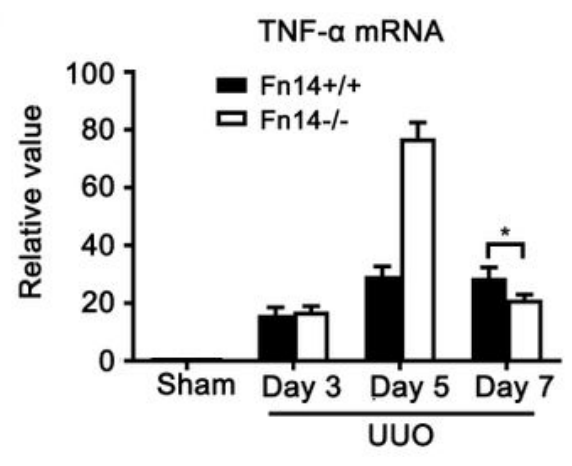

b

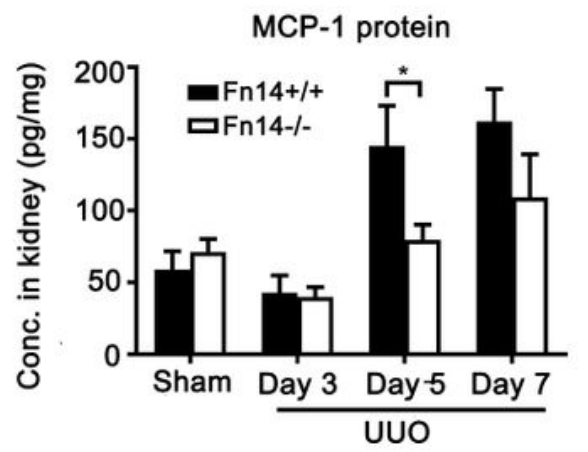

d

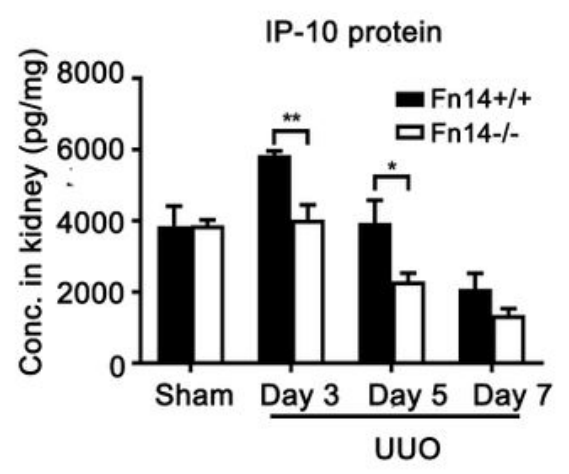

f

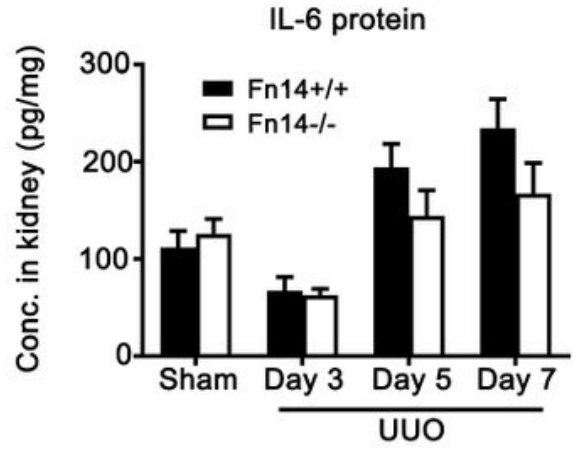

h

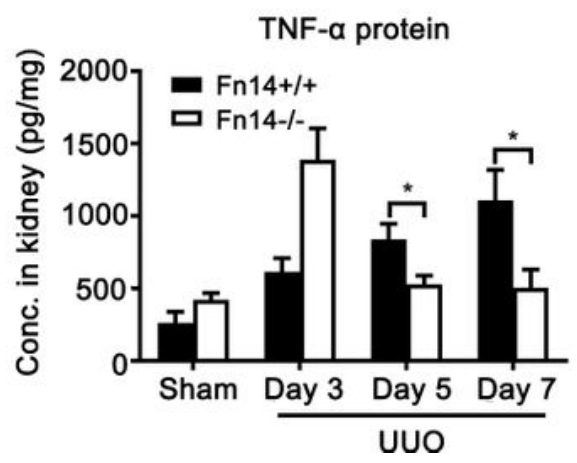

Figure 2

Fn14 deficiency reduced inflammatory cytokine production in the kidneys. UUO was surgically induced in both wild-type and Fn14-deficient mice. qRT-PCR and ELISA were used to determine the mRNA and protein expression levels of MCP-1 (a, b), IP-10 (c, d), IL-6 (e, f), and TNF-a $(g, h)$ in fresh kidney tissues. Number of mice $=5$. ${ }^{*} P<0.05, * \star P<0.01$. 
a

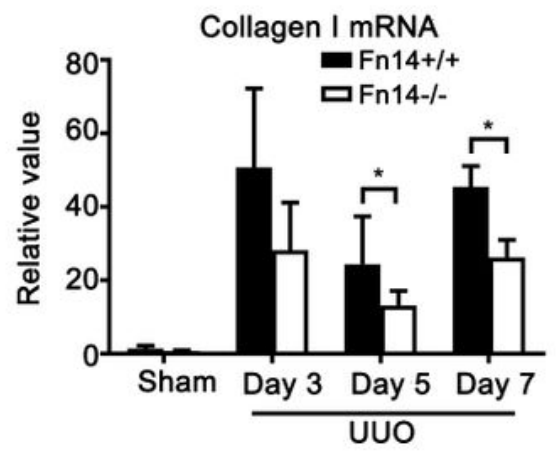

C

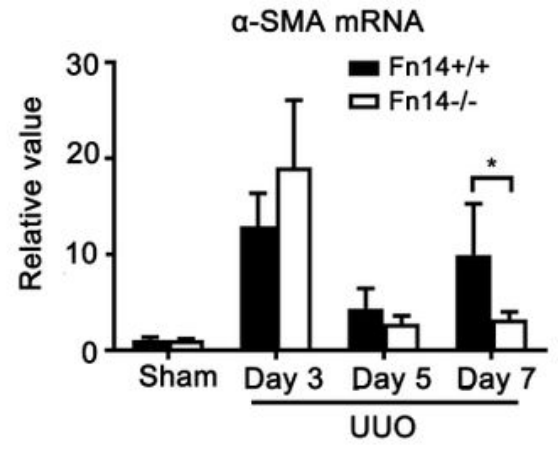

b

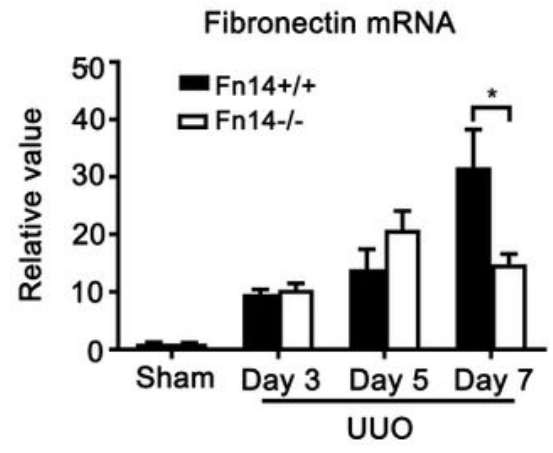

d

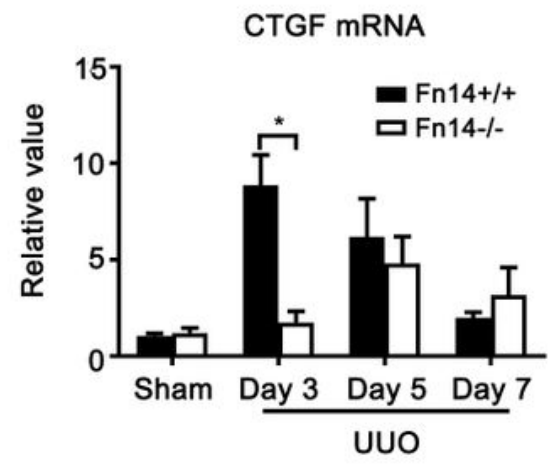

e

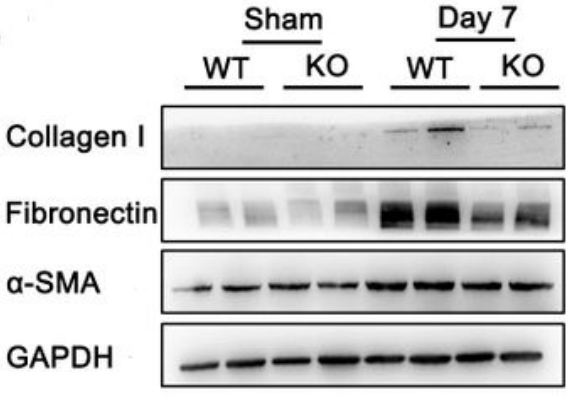

g $\begin{array}{lll}\text { Day } 3 & \text { Day } 5 & \text { Day } 7\end{array}$

$\mathrm{h}$
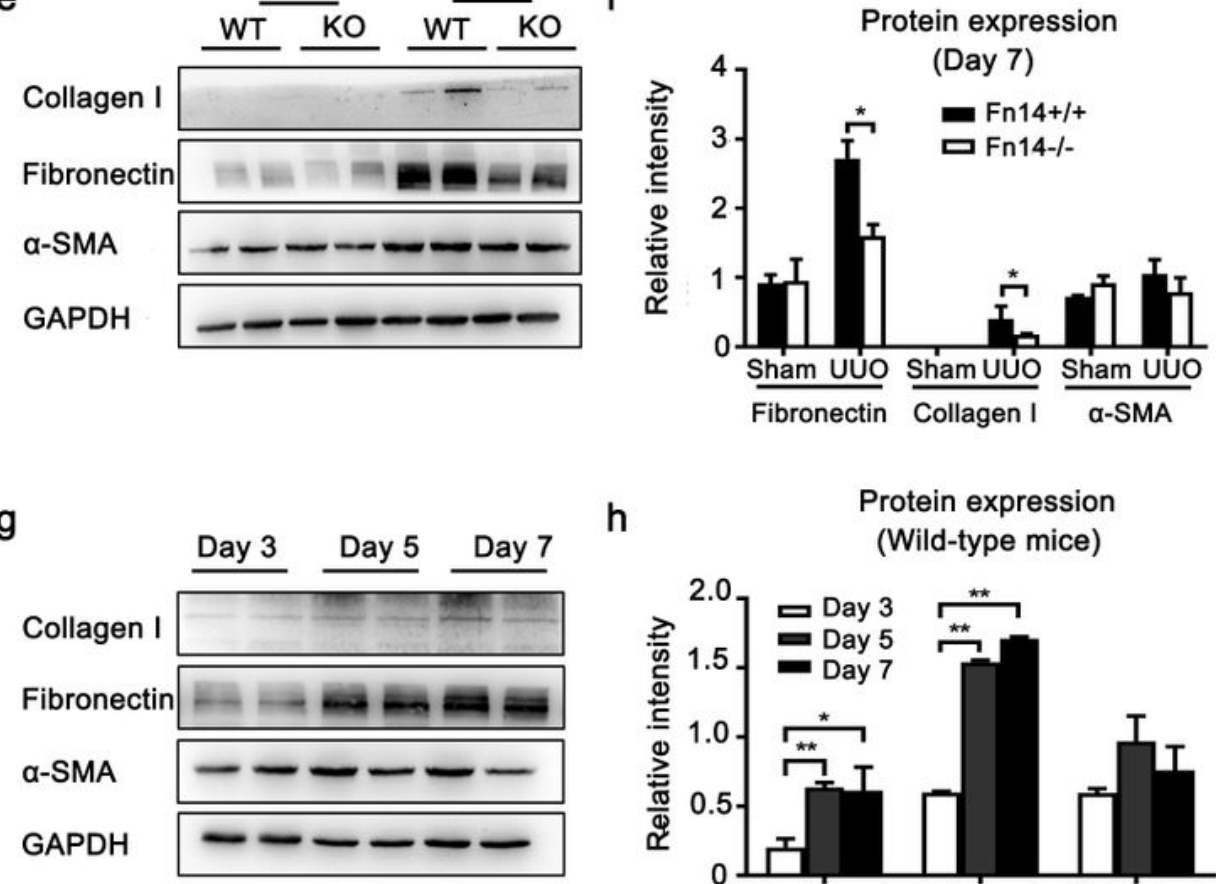

Protein expression

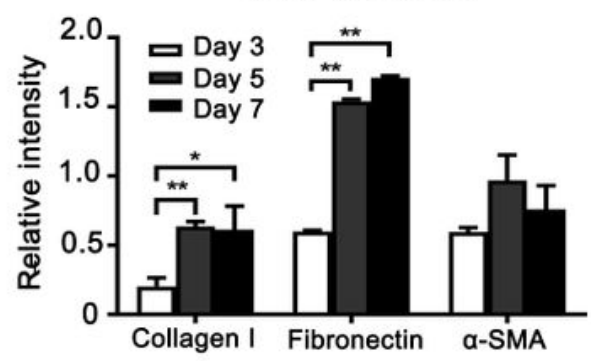

Figure 3

Fn14 deficiency decreased the expression of profibrotic factors and myofibroblast activation markers. UUO was surgically induced in both wild-type and Fn14-deficient mice. $(a-d)$ qRT-PCR was used to determine the mRNA expression levels of collagen I, fibronectin, a-SMA, and CTGF in kidney tissue. (e, f) Western blotting was used to detect collagen I, fibronectin, and a-SMA in fresh tissue. $(\mathrm{g}, \mathrm{h})$ Western 
blotting revealed these proteins in fresh tissue from wild-type mice. Representative images are shown. Number of mice $=5 .{ }^{*} \mathrm{P}<0.05, * * \mathrm{P}<0.01$.

a

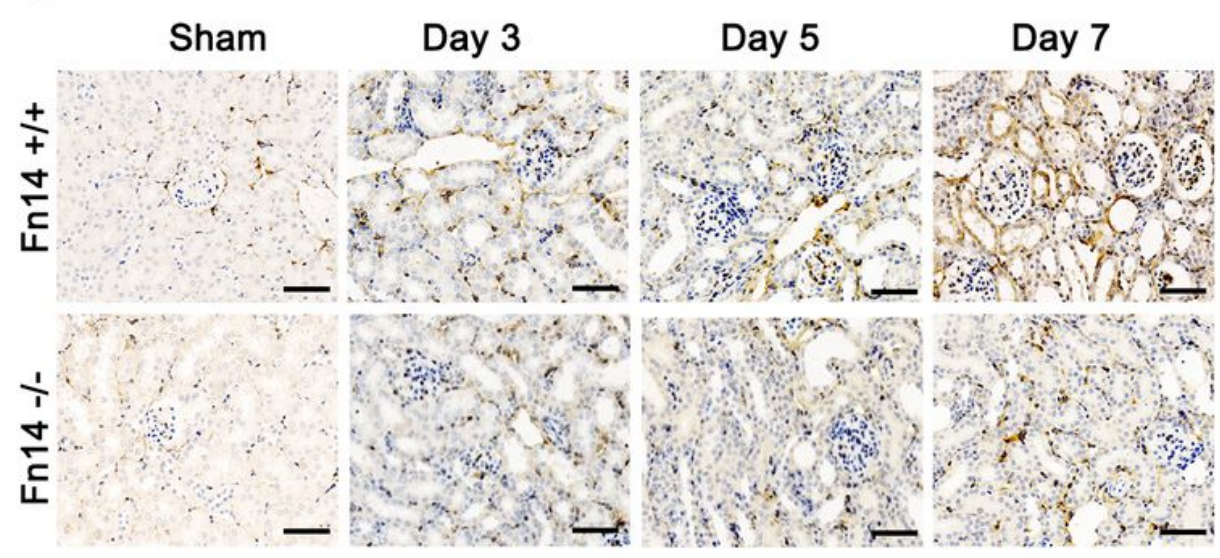

C

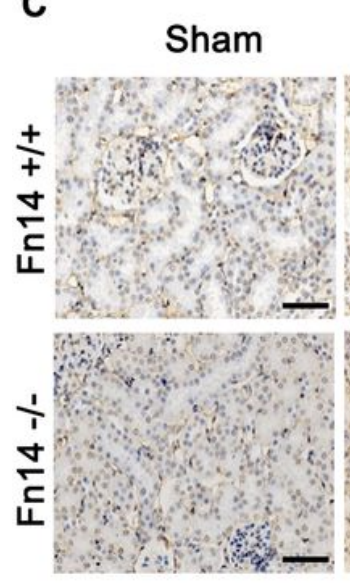

e

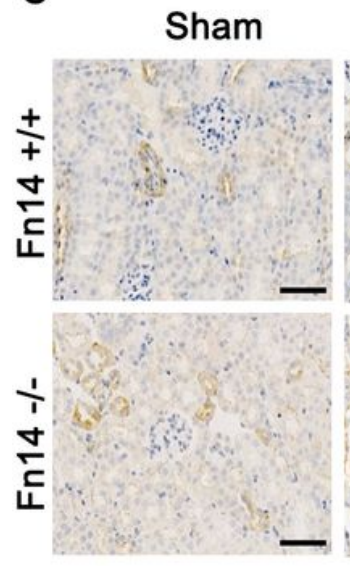

Day 3

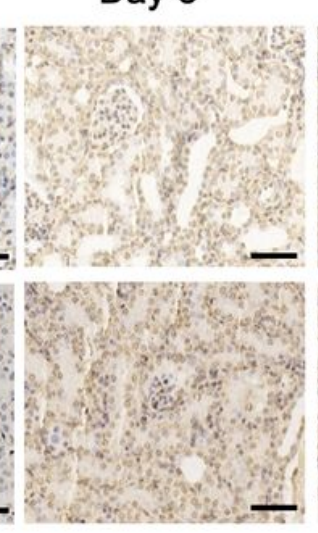

Day 3

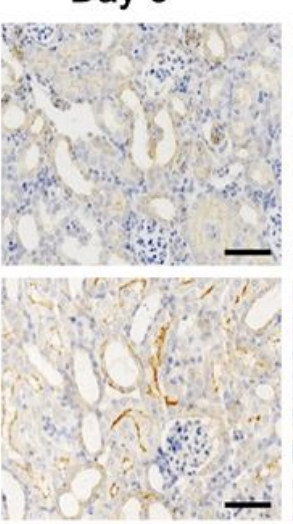

Day 5

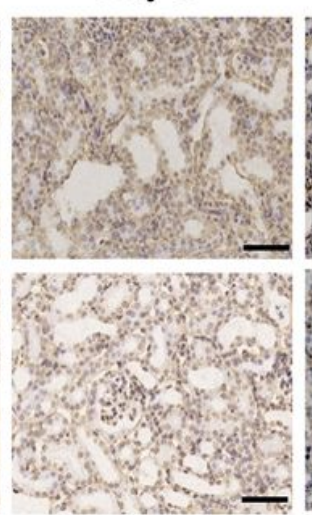

Day 5

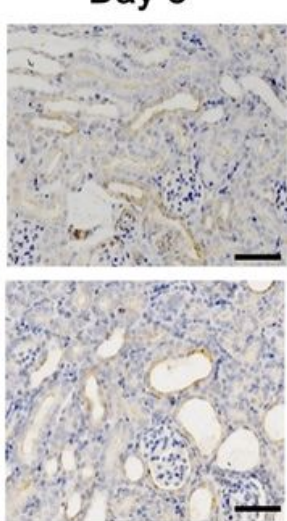

b

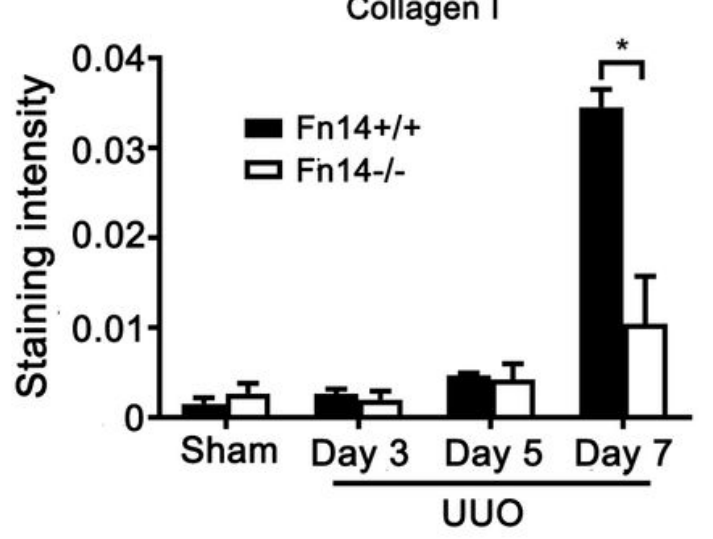

d

Day 7
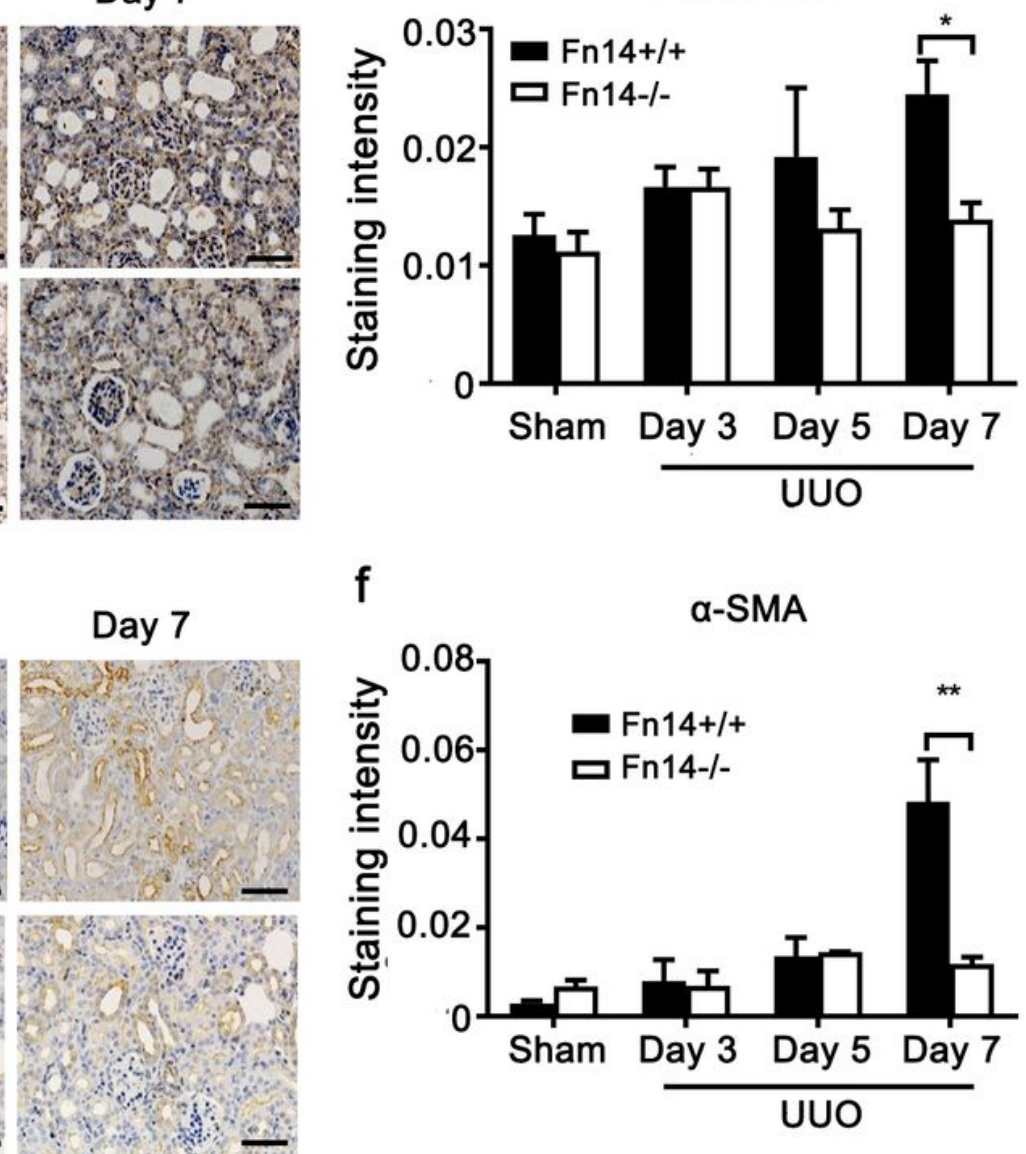

f

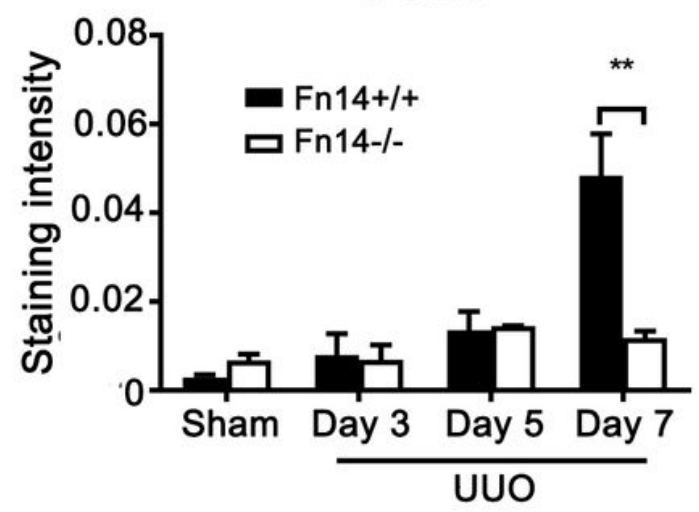

Figure 4

Fn14 deficiency attenuated the extracellular matrix formation. UUO was surgically induced in both Fn14deficient and wild-type mice. Immunohistochemically stained tissue sections showing collagen I $(a, b)$, fibronectin (c, d), and a-SMA (e, f) expression. Intensities of the stained sections were analyzed using 
Image-Pro Plus 6.0 software. Representative images are shown. Number of mice $=5$. Bar $=50 \mu \mathrm{m} .{ }^{*} \mathrm{P}<$ $0.05, * * \mathrm{P}<0.01$.

a

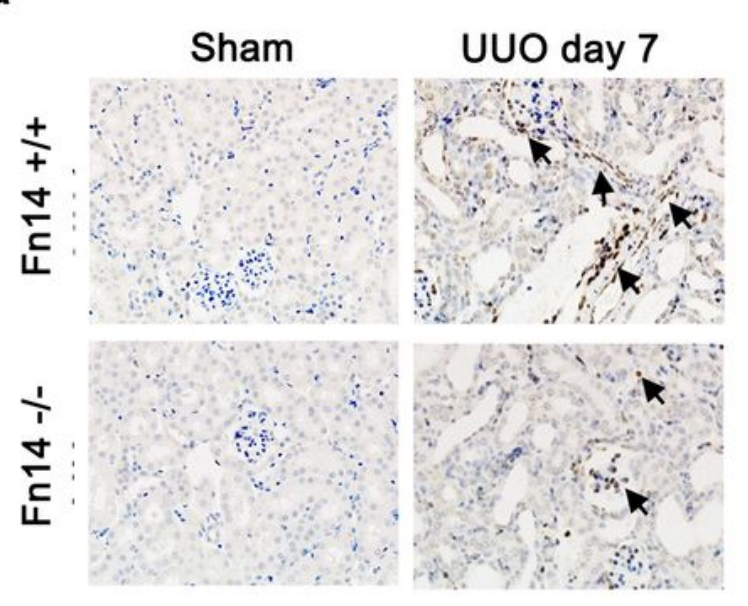

C

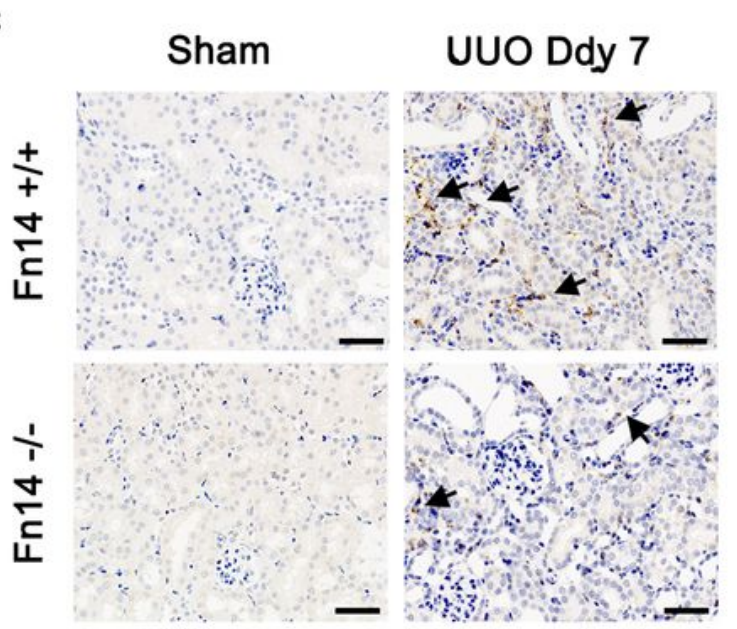

e

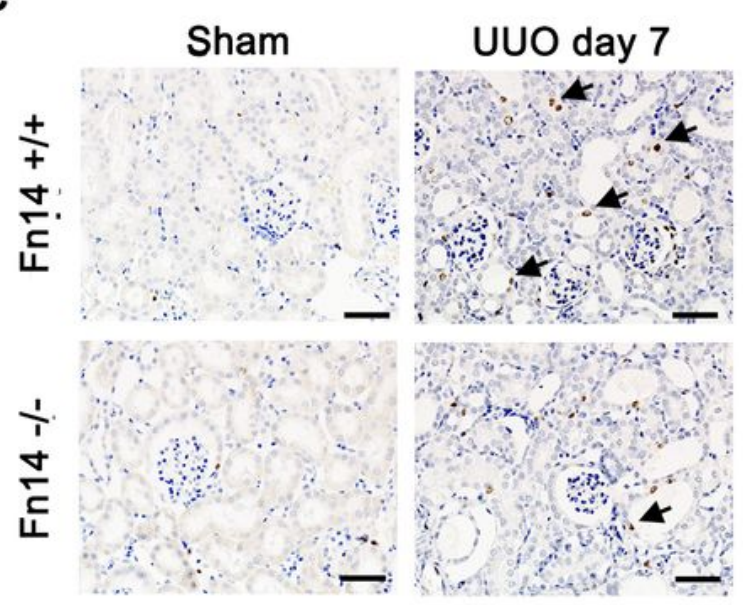

b

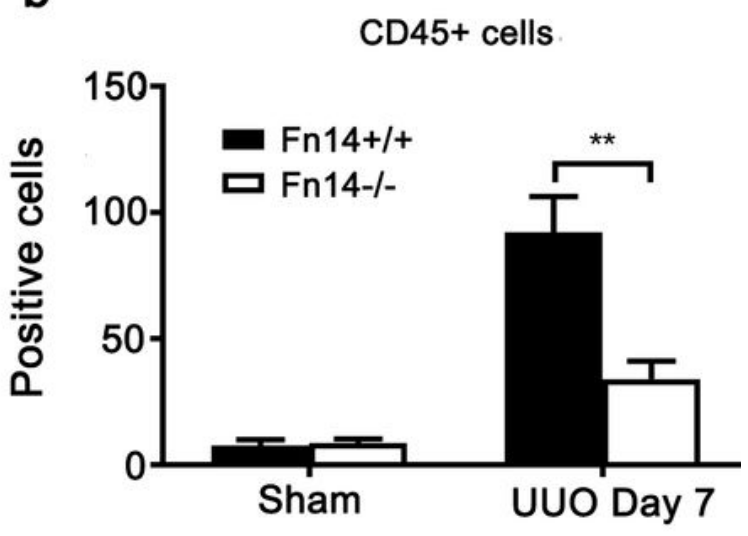

d

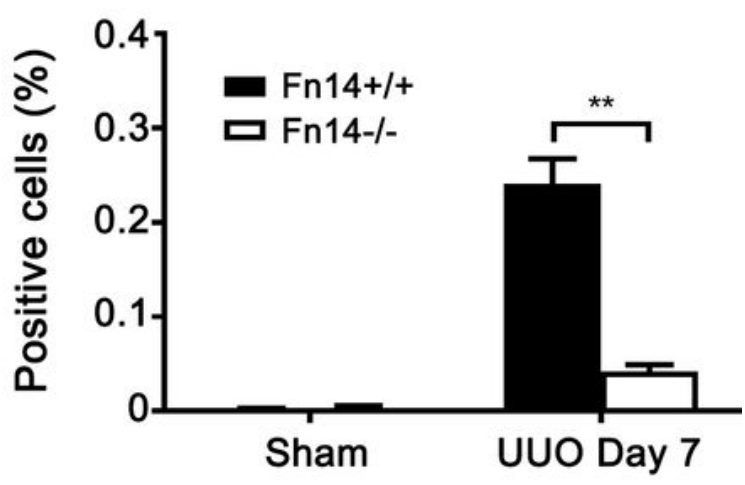

f

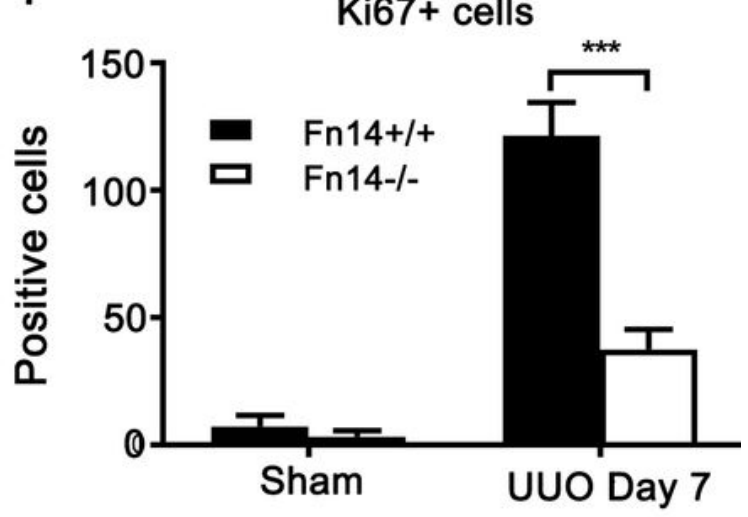

Figure 5

Fn14 deficiency inhibited inflammatory cell infiltration. UUO was surgically induced in both wild-type and Fn14-deficient mice. Immunohistochemistry showing CD45+ leukocytes (a, b), F4/80+ macrophages (c, 
d), and Ki67+ proliferative cells (e,f) in the kidneys on day 7. Representative images are shown. Arrows indicate positive cells. Number of mice $=5$. Bar $=50 \mu \mathrm{m}$. ${ }^{\star \star} P<0.01, * \star \star P<0.001$.

a

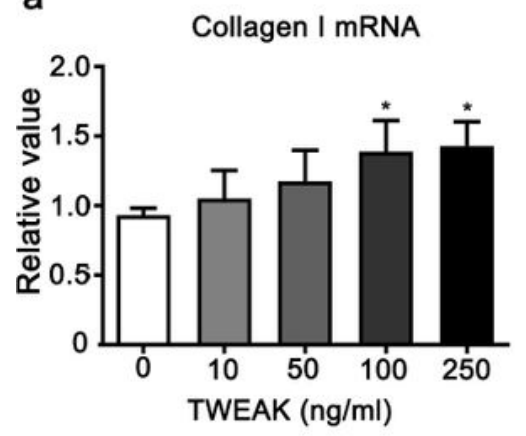

b

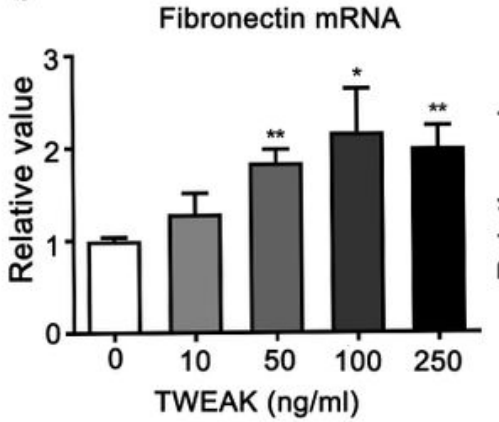

C

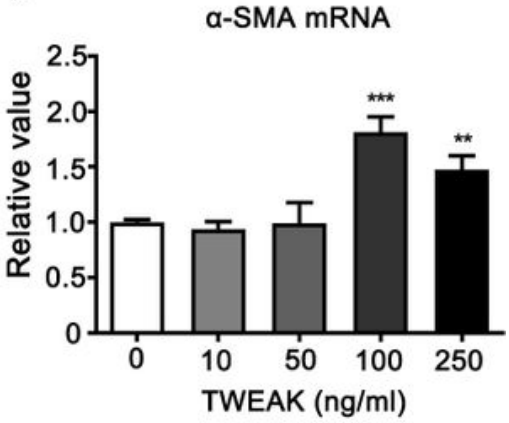

d

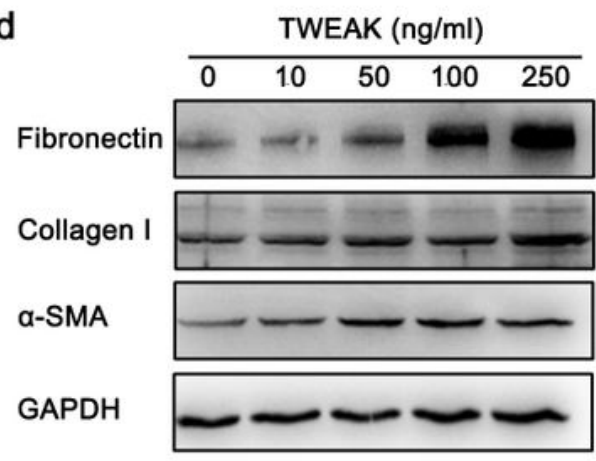

f

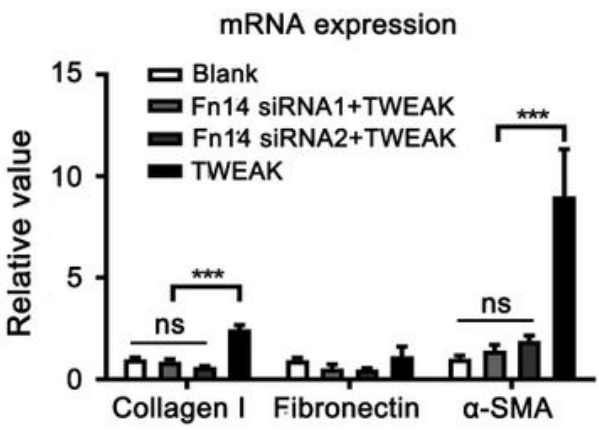

g

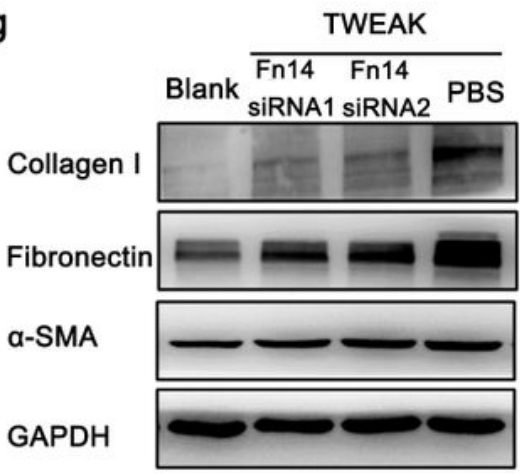

e

Protein expression

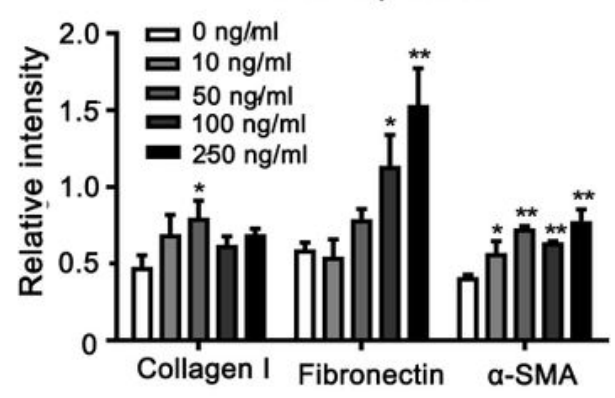

$\mathrm{h}$

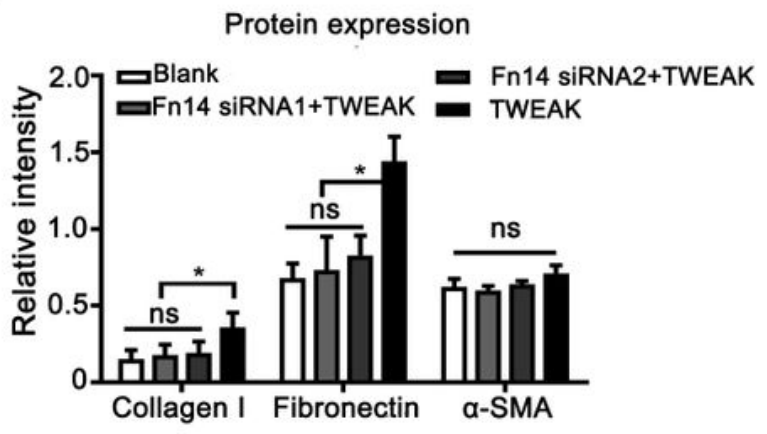

Figure 6

TWEAK promoted profibrotic factor production in HK2 cells. HK2 cells were cultured in vitro and stimulated with recombinant TWEAK (0-250 ng/ml) for $24 \mathrm{~h}$. (a-c) mRNA expression levels of collagen I, fibronectin, and a-SMA were assessed in cells via qRT-PCR. $(d, e)$ Collagen I, fibronectin, and a-SMA 
protein expression levels were determined in cell lysates via Western blotting. (f) qRT-PCR was used to determine the mRNA expression levels in cells after siRNA transfection. $(\mathrm{g}, \mathrm{h})$ Western blotting was used to determine the proteins in cell lysates transfected with siRNA. In (f-h), the TWEAK concentration was $100 \mathrm{ng} / \mathrm{ml}$. Data were obtained from three independent experiments. Representative images are shown in (a-d). ${ }^{*} P<0.05,{ }^{*} \mathrm{P}<0.01,{ }^{* \star *} \mathrm{P}<0.001$, compared with the blank group. ns, not significant.

a

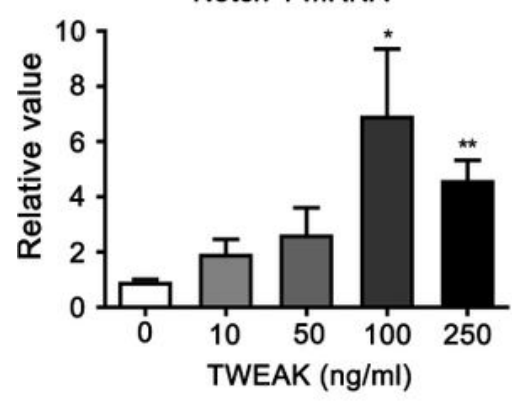

C

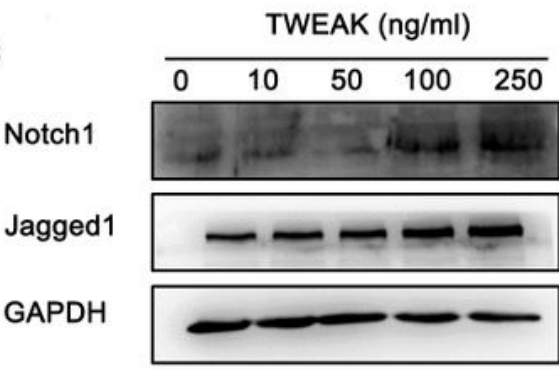

e

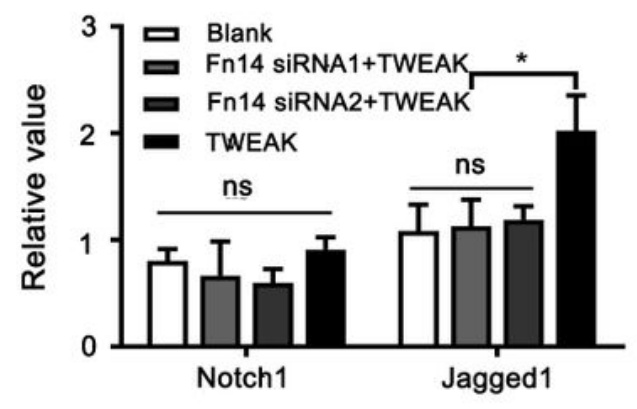

b
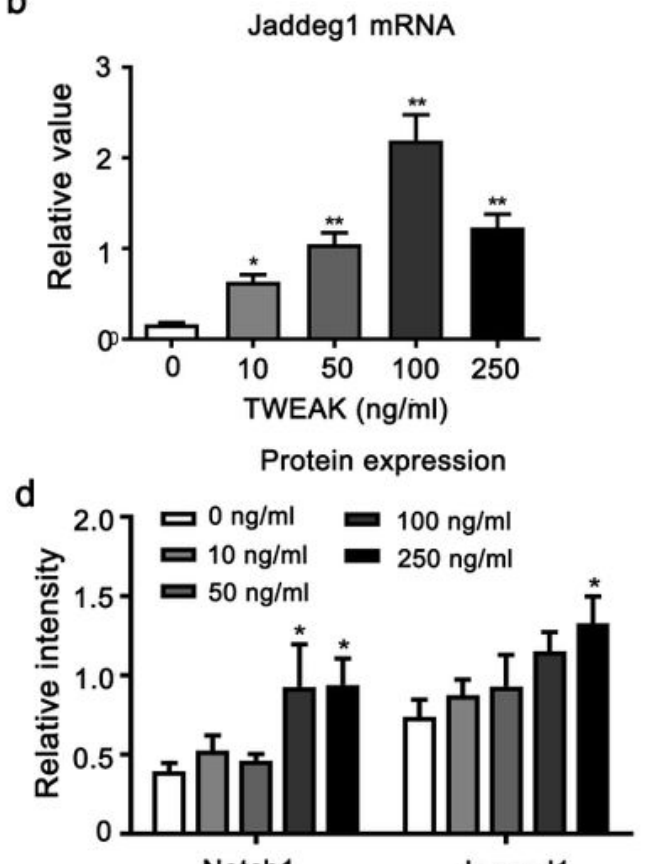

Notch1 f

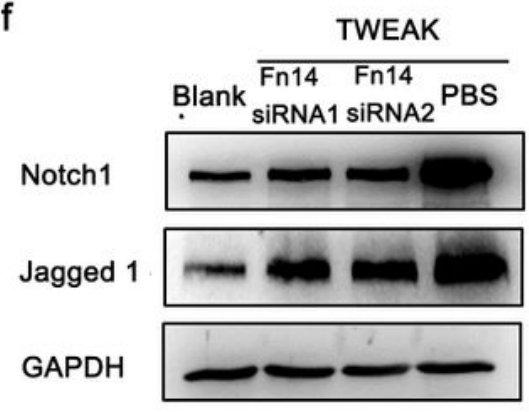

g

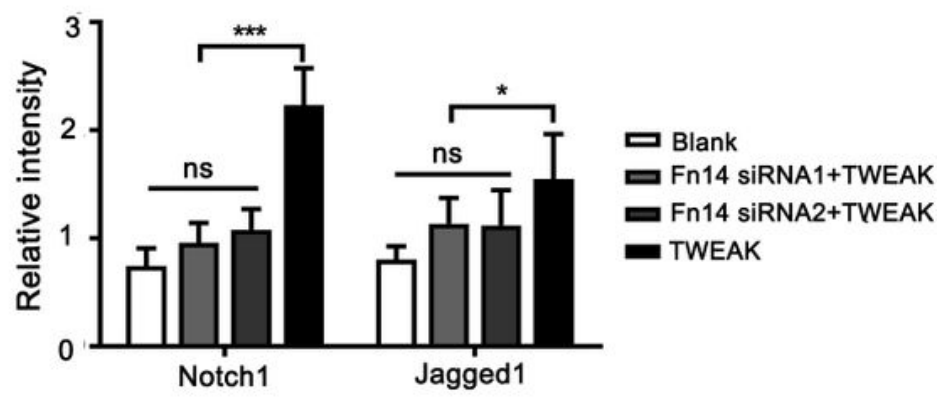

Figure 7 
TWEAK enhanced the Notch1/Jagged1 expression in HK2 cells. HK2 cells were cultured in vitro and received $24 \mathrm{~h}$ stimulation with recombinant TWEAK $(0-250 \mathrm{ng} / \mathrm{ml}) .(\mathrm{a}, \mathrm{b})$ Notch1/Jagged1 mRNA expression levels were assessed in cells via qRT-PCR. (c, d) Western blotting revealed their proteins in the cell lysates. (e) qRT-PCR was used to assess the mRNA expression levels in cells after Fn14 siRNA transfection. $(f, g)$ Western blotting determined their proteins in cells transfected with Fn14 siRNA. In (e$\mathrm{g})$, the TWEAK concentration was $100 \mathrm{ng} / \mathrm{ml}$. Data were obtained from three independent experiments. Representative images are shown. In $(a, b, d),{ }^{*}<0.05$, ${ }^{*} P<0.01$, compared with the blank group. In $(\mathrm{g}), * \star * \mathrm{P}<0.001 . \mathrm{ns}$, not significant.

a

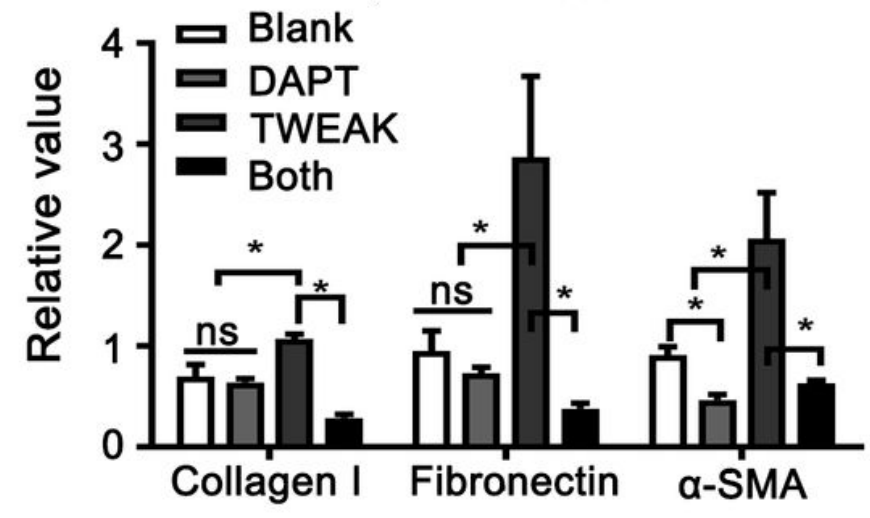

C
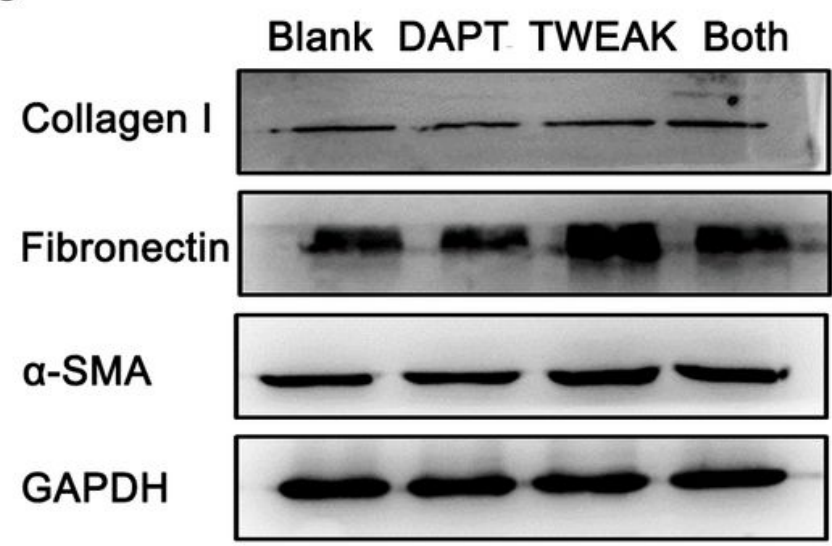

e

Blank DAPT TWEAK Both

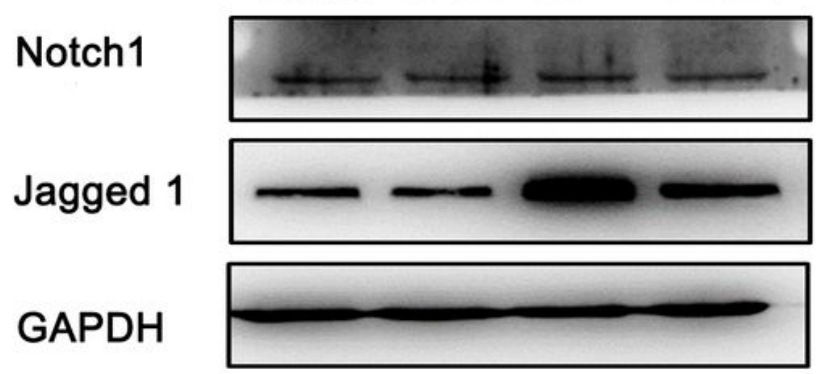

b mRNA expression

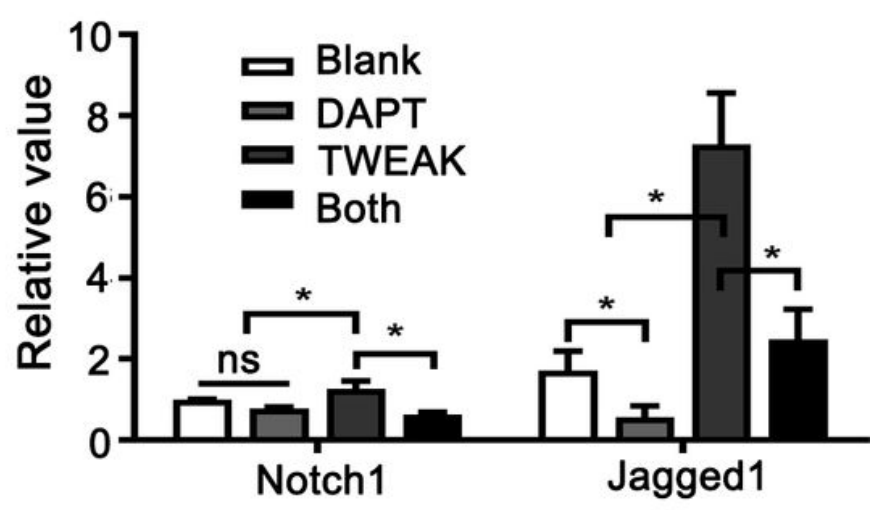

d

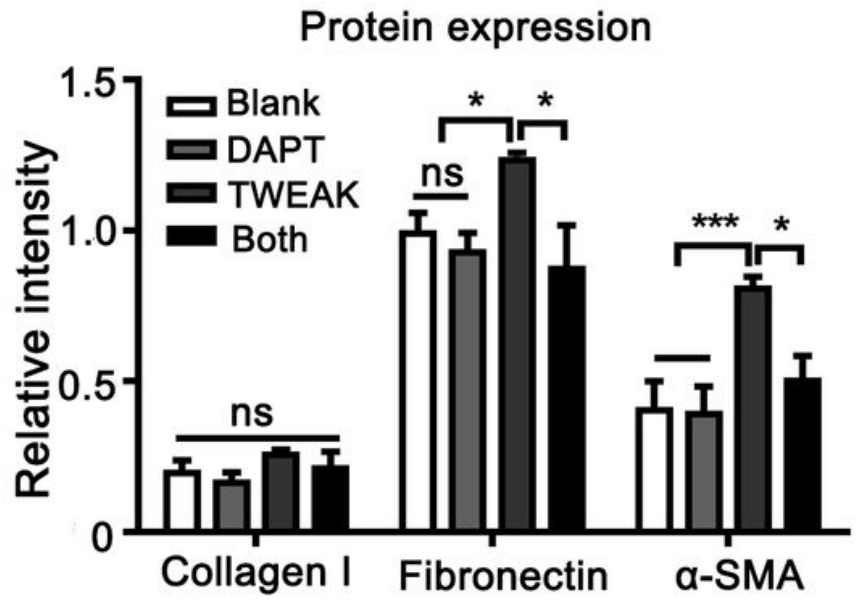

f

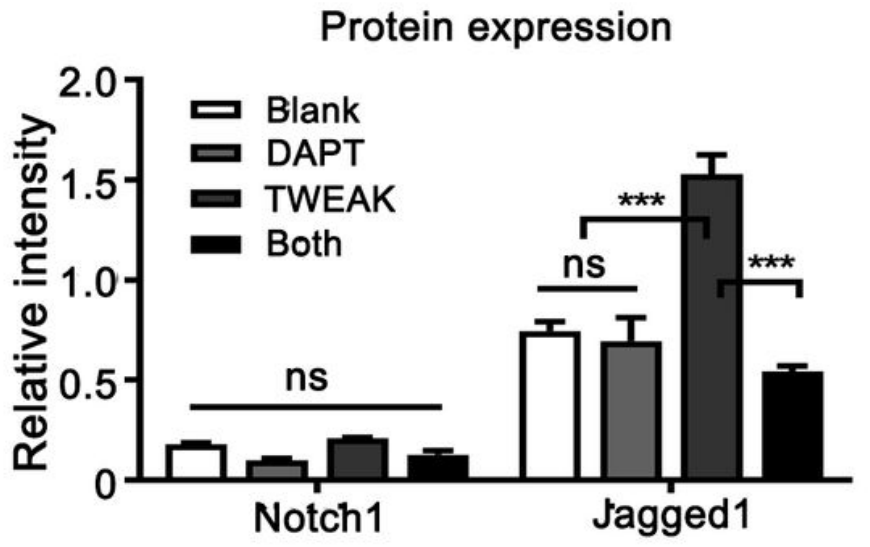

Figure 8 
The Notch1/Jagged1 signal inhibitor, DAPT, mitigated the effect of TWEAK on HK2 cells. HK2 cells were cultured in vitro and stimulated with TWEAK $(100 \mathrm{ng} / \mathrm{ml})$ for $24 \mathrm{~h}$. Some cells were pretreated with DAPT $(200 \mathrm{nM})$ for $1 \mathrm{~h} .(\mathrm{a}, \mathrm{b})$ The mRNA expression levels of profibrotic factors and Notch1/Jagged 1 were assessed in cells via qRT-PCR. (c-f) The protein expression levels of these molecules were determined via Western blotting. Data were obtained from three independent experiments. Representative images are shown. $* P<0.05, * \star \star P<0.001$. ns, not significant.

\section{Supplementary Files}

This is a list of supplementary files associated with this preprint. Click to download.

- FigureS1.pdf

- Figures2.TIF

- FigureS3.TIF

- Figures4.TIF 